\title{
COMPARISON BETWEEN TRADITIONAL RESISTANCE EXERCISE AND VARIABLE RESISTANCE WITH ELASTIC BANDS IN ACUTE VERTICAL JUMP PERFORMANCE
}

original paper

( ) University School of Physical Education in Wroclaw

DOI: https://doi.org/10.5114/hm.2021.103287

\section{DOUGLAS POPP MARIN ${ }^{1,2}$, TODD ANTHONY ASTORINO ${ }^{3}$, ALLAN IGOR SILVA SERAFIM ${ }^{2}$, CHRISTIANO BERTOLDO URTADO ${ }^{4}$, JONATO PRESTES ${ }^{5}$, LUIS FELIPE TUBAGI POLITO ${ }^{6}$, ROSEMARI OTTON $^{1}$}

\author{
${ }^{1}$ Interdisciplinary Post-Graduate Program in Health Science, CBS, Cruzeiro do Sul University, São Paulo, Brazil \\ ${ }^{2}$ Graduation Program in Physical Education, Methodist University of São Paulo, São Bernardo do Campo, Brazil \\ ${ }^{3}$ Department of Kinesiology, California State University San Marcos, San Marcos, USA \\ ${ }^{4}$ Graduation Program on Physical Education, Federal University of Maranhão, Sao Luis, Brazil \\ ${ }^{5}$ Graduation Program on Physical Education, Catholic University of Brasilia, Brasilia, Brazil \\ ${ }^{6}$ Instituto Israelita de Ensino e Pesquisa do Hospital Albert Einstein, São Paulo, Brazil
}

\begin{abstract}
Purpose. Resistance exercise (RE) with variable resistance (VR) may be an interesting strategy to increase strength and power in several sports activities. The present study aimed to compare the effect of RE vs. VR exercise in the back squat on subsequent jump performance at different time points.

Methods. Nine male elite handball athletes (age: $21.4 \pm 2.1$ years; body fat percentage: $10.5 \pm 4.2 \%$ ) undergoing in-season training participated in the study. They randomly completed 3 sets of 5 back squat repetitions at $85 \%$ of predicted one-repetition maximum (1RM) with 3-minute rests between sets. They performed RE back squat alone or VR back squat by combining $55 \%$ of 1 RM using standard weight plates and $29.7 \pm 2.0 \%$ of the athlete's 1RM in fully erect position (starting position) with elastic band resistance. Countermovement jump (CMJ) test was applied at baseline, immediately after the conditioning activity (within 15 seconds), and every 2 minutes after the protocols (2, 4, 6, and 8 minutes).

Results. There was a very large $(5.8 \%, p=0.02, E S=1.53)$ increase in CMJ performance 2 minutes after VR back squat, while no significant changes were observed after the completion of RE back squat. Moreover, there was a large $(p=0.04$, $E S=1.27$ ) increase in peak power after VR exercise.

Conclusions. CMJ performance and peak power output can be potentiated after 2 minutes of recovery following the completion of back squat exercise combining RE plus elastic bands, reinforcing the practical applicability of VR exercise. Key words: jump performance, power output, sports performance, post-activation potentiation, resistance training

\section{Introduction}

Performance and success in many sports rely on the ability to generate adequate strength, power, and speed in movements requiring jumping, throwing, and change of direction [1-3]. Therefore, new strategies to increase muscle power development and resultant athletic performance have been identified not only by athletes and coaches, but also by the scientific community.

Research has shown that traditional resistance training performed with heavy loads promotes increas-

es in power by targeting mainly the force component of the power equation $($ power $=$ force $\times$ velocity) $[1,2]$. On the other hand, unloaded activities such as unloaded vertical jump or ballistic exercises performed with lighter loads are commonly used to achieve higher movement velocities or accelerations, and rate of force development to maximize power production [2]. Thus, the application of training methods combining both higher and lower loads in one training session may save time and additionally produce superior improvements in power production $[4,5]$. This coupling is
\end{abstract}

Correspondence address: Douglas Popp Marin, Cruzeiro do Sul University, Rua Regente Feijó, 1295, 03342-000,

São Paulo - SP, Brazil, e-mail: douglas.marin@metodista.br

Received: November 1, 2019

Accepted for publication: May 17, 2020

Citation: Marin DP, Astorino TA, Serafim AIS, Urtado CB, Prestes J, Polito LFT, Otton R. Comparison between traditional resistance exercise and variable resistance with elastic bands in acute vertical jump performance. Hum Mov. 2021;22(4):28-35; doi: https://doi.org/10.5114/hm.2021.103287. 
referred to as complex training, which consists of alternating biomechanically similar heavy resistance exercise (RE) with plyometric or power exercises in the same workout [6].

The neurophysiological mechanisms that support the application of complex training have been attributed to post-activation potentiation (PAP). Significant improvements in sprinting [7] and vertical jump performance $[4,8-10]$ have been reported after the completion of a conditioning activity $[8,11]$. The aim of complex training is to utilize the PAP effect to improve subsequent power performance, and potentially induce a greater chronic adaptive response [4-6].

Heavy back squat ( $\geq 85 \%$ of one-repetition maximum [1RM]) exercise is commonly investigated to induce the efficacy of PAP, and its use has been reported to improve subsequent performance in trained individuals and athletes $[8,12]$. Conversely, some studies exhibited no difference in vertical jump performance when preceded by back squats $[13,14]$.

Different factors can influence the PAP response after a conditioning activity. Seitz and Haff [12] reported that the magnitude of PAP is modulated by the inherent strength and training status of the individuals, the type of conditioning activity, the rest period between the conditioning activity and subsequent performance, and the number of sets and load expressed as percent of $1 \mathrm{RM}$ of the conditioning activity. Therefore, the type of conditioning activity and the time course of the PAP response must be considered for programming complex training sessions.

Previous research documented a time course of PAP effect $>5$ minutes after the use of heavy RE as a conditioning activity $[12,15]$. In a meta-analysis, Gouvêa et al. [16] suggested that a rest interval of 8-12 minutes after a conditioning activity had a beneficial impact on jump height, whereas a rest interval of $0-3$ minutes induced a detrimental effect on jump performance. This acute enhancement of performance is dependent on the balance between fatigue and potentiation [11]. Therefore, longer recovery intervals are required to reduce fatigue onset, and allow PAP to exert potential ergogenic effects on the subsequent exercise bout. However, in the strength and conditioning setting, a prolonged recovery duration required to elicit an effect of PAP on vertical jump performance may be impractical for athletes and coaches to implement.

Variable resistance (VR) training has emerged as another approach to elicit PAP in a shorter recovery interval compared with RE $[17,18]$. One form of VR combines the use of elastic resistance in conjunction with free weight during various REs, including the back squat, deadlift, and bench press [19]. This approach is characterized by an exponential increase in tension applied throughout the range of motion regardless of joint angle [9]. Previous data show that as elastic bands are stretched, greater resistance is applied on a muscle complex $[9,20]$. This differs from traditional RE, in which the load remains relatively constant throughout the range of motion in function of change in velocity [20, 21]. In addition, studies comparing VR with RE have shown significantly higher muscle activity, movement velocity, and external power in the concentric phase of the back squat exercise when using VR $[9,21]$. Previously, Seitz et al. [18] reported that horizontal jump performance was potentiated after only 90 seconds of recovery following performance of back squat using free weights and elastic RE in rugby players.

Therefore, the purpose of this study was to compare the effect of RE vs. VR exercise in the back squat on subsequent jump performance at different time points in handball athletes. It was hypothesized that RE and VR would enhance countermovement jump (CMJ) performance compared with baseline, and VR would promote a PAP effect after a shorter period owing to the greater velocity during the eccentric phase, which is similar to the plyometric actions. These findings apply to the optimization of various training programs to augment muscle force and power in athletes.

\section{Material and methods}

\section{Design}

This study used a randomized crossover design. Initially, the athletes visited the laboratory to become familiar with the experimental procedures, and their $1 \mathrm{RM}$ in the back squat was determined. Subsequently, 2 experimental sessions and a control trial were conducted. The sessions were completed 7 days apart and held at the same time of day (between 09:00 am. and 11:00 a.m.). The participants were informed only at the start of each visit on the condition they would be exposed to. They reported to the laboratory on the morning of testing after having refrained from alcohol, caffeine, and exercise the day before.

\section{Subjects}

Nine male elite handball athletes who were undergoing in-season training participated in the study (Table 1). They were informed about the experimental procedures and possible discomforts associated with the study. The participants had been engaged in a regu- 
Table 1 . Mean $\pm S D$ demographic values for athletes $(n=9)$

\begin{tabular}{lc}
\hline Characteristics & Mean $\pm S D$ \\
\hline Age (years) & $21.4 \pm 2.1$ \\
Body mass (kg) & $90.3 \pm 10.0$ \\
Height (cm) & $187.5 \pm 5.0$ \\
Body fat (\%) & $10.5 \pm 4.2$ \\
Experience (years) & $9.7 \pm 1.8$ \\
Weekly training volume (hours) & 15 \\
Relative 1RM back squat (kg/kg) & $1.8 \pm 0.2$ \\
\hline
\end{tabular}

$1 \mathrm{RM}$ - one-repetition maximum

lar training program consisting of maximal strength and power training for at least 2 years and were able to squat a minimum of $1.5 \times$ their body mass.

\section{Prediction of 1RM in the parallel back squat} and body composition

During the first visit, body mass and height were determined by using a wall-mounted stadiometer (Sanny, Brazil), and body composition was established with a 7-site skinfold technique (Sanny, Brazil) [22].

The athletes completed a standardized 5-minute warm-up comprising light-intensity cycling and 3 sets of static stretching (20 seconds) of muscle groups associated with the back squat and CMJ. Then, they performed a warm-up consisting of 20 repetitions of the back squat with a 20-kg unloaded bar, 10 repetitions at $50 \%$, and 6 repetitions at $90 \%$ of estimated 10RM (athlete's prediction), with a 2-minute rest period between each load.

After a 3-minute rest, the participants performed the maximum number of repetitions possible with an estimated weight based on the athlete's experience during a single set of back squat to predict 1RM [23]. Estimated 1RM comprised the assessment of 5 to less than 10 maximum repetitions until concentric failure, following recommendations previously described [24]. The athletes were instructed to position the feet shoulder-width apart with a slight external foot rotation (ca. $10^{\circ}$ ) and the barbell was placed above the posterior deltoids (high-bar back squat). The subjects were required to perform the back squat until the top of their thighs was parallel to the ground, which was visually assessed by the first author, and received strong verbal encouragement to promote maximal effort. Moreover, the athletes were instructed to perform the concentric phase of each repetition as rapidly as possible, and to control the eccentric phase until the knees were flexed to approximately $90^{\circ}$ [25].
To predict back squat 1RM, the following equation was used, which has been shown to be highly correlated $(r=0.97)$ with tested 1RM [23]:

$$
1 \mathrm{RM}=[(1+0.0333 \times \text { repetitions }) \times \text { repetitions }+
$$

\section{Band tension measurement}

Elastic bands (Body Band LLC, USA) were attached to the bottom of the squat rack with clips and around both sides of the Olympic bar (Cybex, Medway, USA). The resistance provided by the elastic band was measured by using a load cell (SV 100, Miotec, Porto Alegre, Brazil) to equalize the total load applied by each athlete.

\section{CMJ test}

CMJ performance was assessed by using a contact time mat (Jump Test 2.0, Hidrofit Ltda, Belo Horizonte, Brazil), consisting of a mat connected to a digital timer (10 ms). This system has been demonstrated to be reliable for the measurement of flight time [26]. Jump height was determined with the following equation:

$$
\text { jump height }=\left[9.81 \times(\text { flight time })^{2}\right] / 8
$$

The equation by Sayers et al. [27] was used to convert jump height into peak power. Peak power displayed a high test-retest reliability with an interclass correlation coefficient (ICC) value of 0.98 .

The CMJ assessment started with athletes in the upright position; they were instructed to flex their knees approximately $90^{\circ}$ as quickly as possible and then jump as high as possible in the ensuing concentric phase. A minimum of 10-second recovery was allowed between jump trials, and each athlete performed 3 maximal CMJs. The subjects were instructed to maintain their hands on the hips to eliminate the effect of arm swing. They were well familiarized with the testing procedures, and the best $\mathrm{CMJ}$ attempt was recorded for further analysis. CMJ jump height displayed acceptable test-retest reliability with an ICC value of 0.84 .

\section{Experimental sessions}

Initially, the athletes performed a standardized warm-up comprising 5 minutes of light-intensity cycling and 3 sets of static stretching with an emphasis on muscle groups associated with back squat. Subse- 


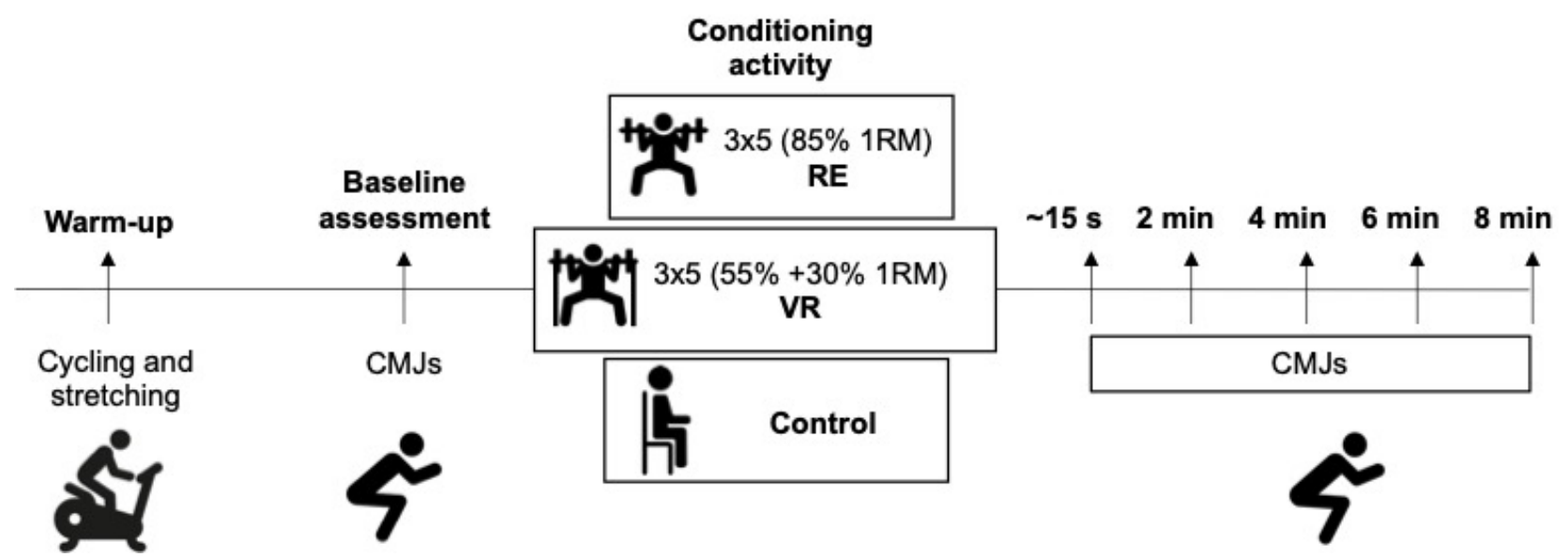

CMJ - countermovement jump, RE - resistance exercise, VR - variable resistance

Figure 1. Schematic representation of the experimental protocol

quently, they were given 3 minutes of passive recovery and completed the baseline CMJ test. Immediately after (within 15 seconds) the conditioning activity (3 sets of 5 back squat repetitions) and every 2 minutes after the PAP protocol (2, 4, 6, and 8 minutes), the participants completed the CMJ test (Figure 1) as previously described [28]. In order to account for the possible fatiguing or potentiating effects from repeated maximal jumps performed after the conditioning activity, a control condition that included repeated performance of CMJ tests without conditioning activity was also completed.

In the VR and RE conditions, the athletes performed 3 sets of 5 back squat repetitions at $85 \%$ of predicted $1 R M$, with 3 minutes of rest between sets. For VR, the intensity was determined by combining $55 \%$ of $1 \mathrm{RM}$ using standard weight plates and $29.7 \pm 2.0 \%$ of the athlete's 1RM in fully erect position (starting position) with elastic band resistance.

We used the following equation to determine the potentiating effect of conditioning activity on subsequent CMJ performance:

$$
\% \text { PAP }=\left[\left(\mathrm{CMJ}_{\mathrm{PAP}}-\mathrm{CMJ}_{\text {BASELINE }}\right) / \mathrm{CMJ}_{\mathrm{PAP}}\right] \times 100
$$

where $\mathrm{CMJ}_{\mathrm{PAP}}$ represents the highest $\mathrm{CMJ}$ measured in each time point after PAP protocol, and $\mathrm{CMJ}_{\text {BASELINE }}$ is the baseline CMJ height.

\section{Statistical analysis}

Statistical analyses were performed with the use of the SPSS software (SPSS Inc., Chicago, USA) and data are presented as mean $\pm S D$. Significance was set at $p<0.05$. Two-way repeated measures ANOVA served to compare the PAP effect across time (5 levels: im- mediately after exercise, 2 minutes, 4 minutes, 6 minutes, 8 minutes) and condition (3 levels: VR, RE, control). ICCs were used to determine the test-retest reliability of CMJ jump height and peak power at baseline. CMJ tests were applied during each testing session. Effects size using Cohen's $d$ was calculated to consider the magnitude of difference in the PAP effect between protocols. Differences were considered trivial at $<0.20$, small at $0.20-0.50$, medium at $0.50-$ 0.80 , large at $0.80-1.30$, or very large at $>1.30$.

\section{Ethical approval}

The research related to human use has complied with all the relevant national regulations and institutional policies, has followed the tenets of the Declaration of Helsinki, and has been approved by the Ethics Committee of the Methodist University of São Paulo.

\section{Informed consent}

Informed consent has been obtained from all individuals included in this study.

\section{Results}

\section{CMJ performance}

Two-way repeated measures ANOVA revealed a significant main effect of time $(\mathrm{F}(5,40)=5.50, p=0.001$, $\left.\eta_{\mathrm{p}}^{2}=0.40\right)$, but no significant condition effect $(p=0.39)$ or time $\times$ condition interaction $(p=0.31)$. There was a nonsignificant time effect for $\mathrm{CMJ}$ performance in the control condition $(p=0.80)$. Pairwise comparisons indicated improvements of 5.8\% in CMJ height ( $p=0.02, E S=1.53$, 'very large') at 2 minutes of recovery in the VR condition compared with pre-intervention. The change in $\mathrm{CMJ}$ performance was 'very 


\section{HUMAN MOVEMENT}

D. Marin et al., Variable resistance and jump performance

large' at 4 minutes $(p=0.18, E S=1.41)$ but did not reach statistical significance. No significant differences were observed in CMJ performance in the RE condition (Table 2).

\section{CMJ peak power}

Two-way repeated measures ANOVA indicated a significant main effect of time $(\mathrm{F}(5,40)=4.22, p=$ $\left.0.04, \eta_{\mathrm{p}}^{2}=0.35\right)$, but no significant condition effect $(p=0.18)$ or time $\times$ condition interaction $(p=0.47)$. There was a nonsignificant time effect for CMJ performance in the control condition $(p=0.88)$. Pairwise comparisons revealed a significant increase in CMJ peak power ( $p=0.02, E S=1.27$, 'large') at 2 minutes of recovery in the VR condition compared with pre-in- tervention. No significant differences were observed in $\mathrm{CMJ}$ peak power in the RE condition (Table 2).

\section{PAP response}

Two-way repeated measures ANOVA indicated a significant main effect of time $(\mathrm{F}(4,32)=3.88, p=$ $\left.0.01, \eta_{\mathrm{p}}^{2}=0.33\right)$ and time $\times$ condition interaction $\left(\mathrm{F}(8,64)=2.09, p=0.05, \eta_{\mathrm{p}}^{2}=0.21\right)$, but no significant condition effect ( $p=0.27)$. At 2 minutes of recovery, pairwise comparisons showed that the PAP effect was significantly larger $(+4.9 \%, p=0.02, E S=$ 0.70 , 'medium') in the VR as compared with the control condition. No PAP effect was observed in response to RE across time (Figure 2).

Table 2. Countermovement jump performance and peak power across time points after the free weight, variable resistance, and control conditions

\begin{tabular}{llcccccc}
\hline Variable & Condition & Baseline & Ca. $15 \mathrm{~s}$ & $2 \mathrm{~min}$ & $4 \mathrm{~min}$ & $6 \mathrm{~min}$ & $8 \mathrm{~min}$ \\
\hline \multirow{2}{*}{ CMJ } & VR & $41.4 \pm 2.5$ & $41.4 \pm 3.1$ & $43.8 \pm 3.0^{*}$ & $43.8 \pm 3.9$ & $42.8 \pm 4.3$ & $41.6 \pm 3.9$ \\
$(\mathrm{~cm})$ & $\mathrm{RE}$ & $41.5 \pm 4.2$ & $39.7 \pm 4.7$ & $42.0 \pm 3.6$ & $41.8 \pm 3.6$ & $41.9 \pm 4.5$ & $40.7 \pm 4.3$ \\
& Control & $44.1 \pm 3.6$ & $45.2 \pm 3.2$ & $45.3 \pm 3.3$ & $44.7 \pm 3.3$ & $44.9 \pm 2.5$ & $45.0 \pm 3.9$ \\
\hline \multirow{2}{*}{ Peak power } & VR & $6683.2 \pm 451.3$ & $6674.9 \pm 409.9$ & $6798.6 \pm 419.7^{*}$ & $6793.6 \pm 379.9$ & $6748.3 \pm 407.3$ & $6669.4 \pm 397.3$ \\
$(\mathrm{~W})$ & $\mathrm{RE}$ & $6691.5 \pm 477.7$ & $6587.7 \pm 431.1$ & $6704.8 \pm 497.2$ & $6693.3 \pm 526.9$ & $6706.6 \pm 491.4$ & $6633.8 \pm 458.6$ \\
& Control & $6822.9 \pm 414.8$ & $6876.4 \pm 451.6$ & $6885.2 \pm 431.4$ & $6857.6 \pm 453.4$ & $6873.1 \pm 451.9$ & $6885.2 \pm 406.5$ \\
\hline
\end{tabular}

CMJ - countermovement jump, VR - variable resistance, $\mathrm{RE}$ - resistance exercise

* significantly different from baseline value within condition $(p<0.05)$

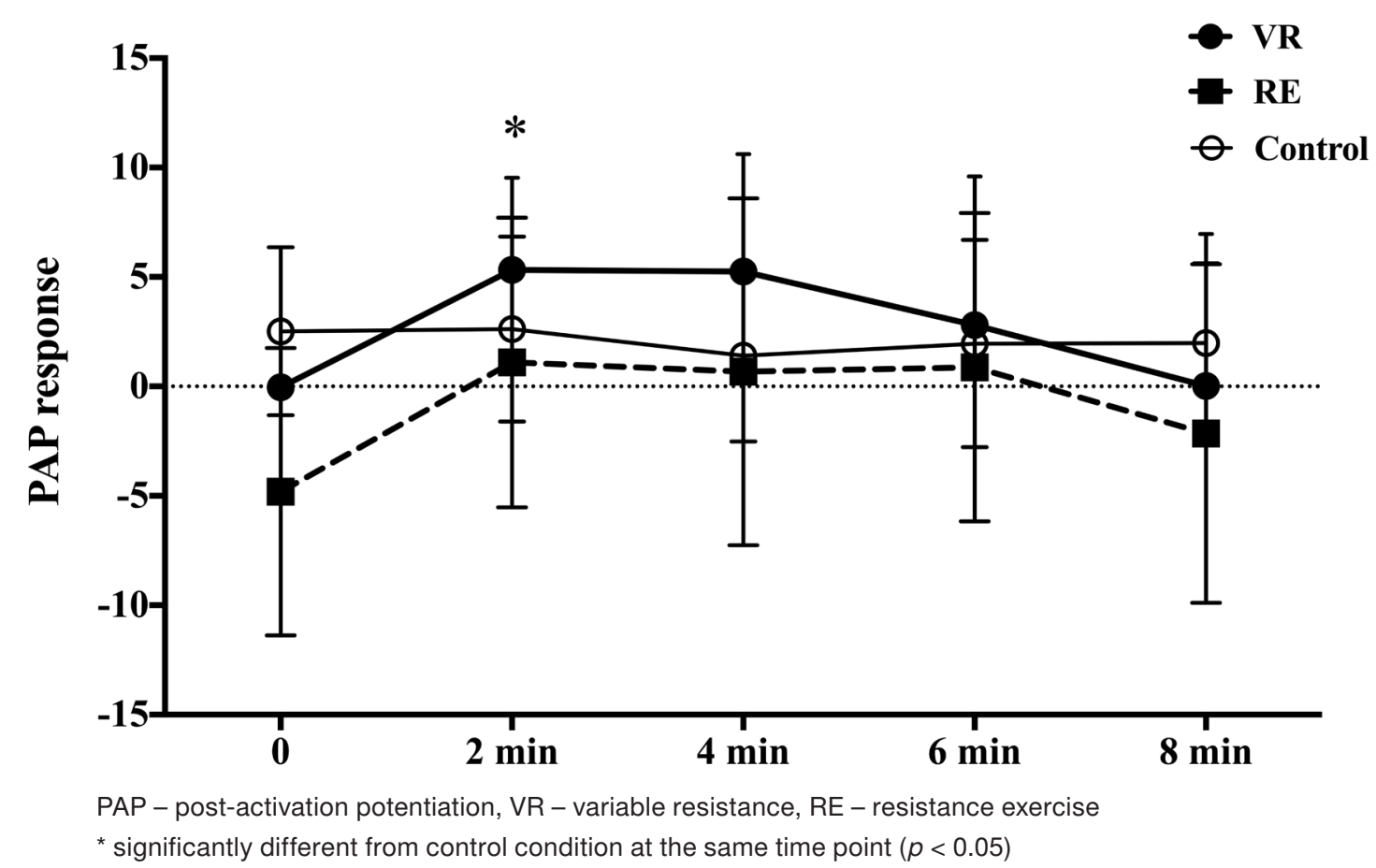

Figure 2. Changes in PAP response during the recovery period after variable resistance, resistance exercise, and control condition (expressed as mean $\pm S D$ ) 


\section{Discussion}

Strength and conditioning professionals seek effective training strategies to optimize the chronic response to training and augment exercise performance in their athletes. Previous results show that, yet it is equivocal whether RE or VR exercise is superior to induce PAP and improve subsequent power production. The PAP effects depend on the appropriate balance between neuromuscular potentiation and fatigue, which emphasizes identification of the optimal balance of volume, intensity, conditioning activity, and recovery time to augment this intervention [14]. In the present study, we compared the effect of RE and VR as a conditioning activity on PAP and subsequent CMJ performance. Our results in elite handball athletes showed a very large (maximal increase in PAP of $5.8 \%, E S=1.53$ ) increase in CMJ performance 2 minutes after VR back squat, while no significant changes were observed after the completion of RE back squat.

Examining PAP response in rugby athletes, Baker [29] showed that 2 heavy box squats combined with elastic bands increased peak power output by $6-7 \%$ during the jump squats after 90 seconds of recovery. Scott et al. [17] reported an acute increase in CMJ performance at 30 seconds, but not 90 or 180 seconds, after a single set of back squat ( $70 \%$ of $1 \mathrm{RM})$ with elastic band resistance ( $23 \%$ of $1 \mathrm{RM})$. In another study with rugby players, Seitz et al. [18] determined an increase in horizontal jump performance of $4-5.7 \%$ (ES: 0.58-0.81) after 90 seconds of recovery using back squat VR ( $85 \%$ of 1 RM using $70 \%$ of free weight plus $15 \%$ of elastic band resistance). The authors observed that stronger athletes (back squat $>1.85 \times$ body mass) presented a larger PAP response (6.5\%) compared with those who were less trained (3.2\%). These data support our findings, which indicated similar magnitudes of increase in jump performance $(5.8 \%$, $E S=1.53$ ) in strong handball athletes (back squat = $1.8 \times$ body mass). In fact, stronger athletes are more able to exhibit PAP response after heavy conditioning activities [14].

PAP can occur earlier if less fatigue is produced by the preceding conditioning activity [14]. We observed the mean peak PAP response on CMJ performance occurred at approximately 2 minutes; however, 3 of the 9 athletes expressed the peak 4 minutes after VR back squat. Taking into account the current recommendation of 8-12 minutes of recovery to optimize PAP response on vertical jump following the completion of traditional RE [16], our data corroborate previous findings supporting the use of VR as a time-effi- cient method to evoke PAP after only 30-120 seconds in well-trained athletes. However, the optimal recovery time should be determined individually since some athletes require more time to dissipate the effects of fatigue.

Several physiological mechanisms have been suggested to explain the enhanced performance after PAP, including an increase in the phosphorylation of myosin light chain, increase in muscle stiffness, and neuromuscular activation [14]. More specifically, the early PAP response observed after VR conditioning activity may be due to higher neural activity and greater total work attained during the lift when compared with traditional RE [9, 21].

Our results indicated a large $(E S=1.27)$ increase in peak power after VR exercise. Studies have highlighted the importance of peak power during CMJ for team sports such as handball which requires high levels of lower-body power output during sprints, jumps, and changes in directions [30]. Therefore, PAP may have the potential to enhance the power output of the $\mathrm{CMJ}$, resulting in a greater acute and chronic training stimulus [6, 11, 31]. The use of VR may enable both high lifting velocities and force production, which may create a favourable stimulus for increasing power output for the next exercise in the complex training session [29].

The obtained outcomes partially refute our first hypothesis as there was a lack of PAP response after $\mathrm{RE}$ conditioning activity. In fact, a close examination of the literature reveals inconsistent findings regarding the PAP response on CMJ performance. For example, Mola et al. [32] and Esformes et al. [33] reported no changes in CMJ height or peak power output after different back squat conditioning activities in soccer players. In contrast, Kilduff et al. [15] demonstrated increases in CMJ height $(+4.9 \%)$ and power output following a heavy back squat in professional rugby athletes.

In the present study, the load equal to $85 \%$ of $1 \mathrm{RM}$ and the number of sets were chosen on the basis of the results of previous experimental [15, 34] and metaanalytic studies [14] in elite athletes. Nevertheless, we suggest that the effects of fatigue induced by RE back squat are equalled those of PAP during the recovery period, and therefore may attenuate any enhancement of CMJ performance. It is possible that VR may be less fatiguing than RE when the same intensity is used [31]. Discrepancies in results across studies can be related to the differences in participants' muscular strength, the duration of the recovery period between the conditioning exercise and the perfor- 
mance test, and the specific intensity of the conditioning exercise $[14,16]$.

The low number of participants $(n=9)$ should be considered a limitation of the present study as it may have reduced our ability to find a significant effect of condition. However, the subjects were elite athletes, quite homogeneous in their physical characteristics and training experience. On the other hand, the inclusion of the control condition in a randomized design is an important strength of the present study.

The lack of equipment and electronic devices (force cells, contact time mat, linear encoder) in clubs and facilities for individual PAP assessment can be considered a limitation for the application of complex training based on the PAP effect.

\section{Practical applications}

VR may be used as a more time-effective method of eliciting PAP in complex training sessions involving CMJ, especially for team sports athletes. The maximal increase in PAP of 5.8\% may create a greater training stimulus over time, and may subsequently augment athletes' long-term training adaptations and sports performance. The ability to use the PAP response elicited by VR conditioning activity during strength and power workouts seems to be feasible to implement by strength and conditioning professionals seeking to enhance the mechanical stimulus associated with explosive training modalities.

\section{Conclusions}

Our results suggest that $\mathrm{CMJ}$ performance and peak power output can be potentiated after 2 minutes of recovery following the completion of back squat exercise combining free weight (55\% of $1 \mathrm{RM})$ plus elastic bands ( $30 \%$ of $1 \mathrm{RM})$, while RE back squat did not change CMJ performance. Future research is required to determine the optimal VR loads to elicit the PAP response, and should consider the individualization of both the recovery period and the load in the conditioning activity.

\section{Acknowledgments}

The authors thank the participants for their effort in completion of this study and the Coordination for the Improvement of Higher Education Personnel (CAPES).

\section{Disclosure statement}

No author has any financial interest or received any financial benefit from this research.

\section{Conflict of interest}

The authors state no conflict of interest.

\section{References}

1. Cormie P, McGuigan MR, Newton RU. Developing maximal neuromuscular power: part 2 - training considerations for improving maximal power production. Sports Med. 2011;41(2):125-146; doi: 10.2165/11538 500-000000000-00000.

2. Haff GG. Training principles for power. Strength Cond J. 2012;34(6):2-12; doi: 10.1519/SSC.0b013e31826 db467.

3. Coratella G, Beato M, Cè E, Scurati R, Milanese C, Schena F, et al. Effects of in-season enhanced negative work-based vs traditional weight training on change of direction and hamstrings-to-quadriceps ratio in soccer players. Biol Sport. 2019;36(3):241-248; doi: 10.5114/biolsport.2019.87045.

4. Bauer P, Uebellacker F, Mitter B, Aigner AJ, Hasenoehrl T, Ristl R, et al. Combining higher-load and lower-load resistance training exercises: a systematic review and meta-analysis of findings from complex training studies. J Sci Med Sport. 2019;22(7):838-851; doi: 10.1016/j.jsams.2019.01.006.

5. Pagaduan J, Schoenfeld BJ, Pojskić H. Systematic review and meta-analysis on the effect of contrast training on vertical jump performance. Strength Cond J. 2019;41(3):63-78; doi: 10.1519/SSC.0000000000000 442.

6. Ebben WP. Complex training: a brief review. J Sports Sci Med. 2002;1(2):42-46.

7. Lima JCB, Marin DP, Barquilha G, Da Silva LO, Puggina EF, Pithon-Curi TC, et al. Acute effects of drop jump potentiation protocol on sprint and countermovement vertical jump performance. Hum Mov. 2011; 12(4):324-330; doi: 10.2478/v10038-011-0036-4.

8. Bauer P, Sansone P, Mitter B, Makivic B, Seitz LB, Tschan H. Acute effects of back squats on countermovement jump performance across multiple sets of a contrast training protocol in resistance-trained men. J Strength Cond Res. 2019;33(4):995-1000; doi: 10.1519/JSC.0000000000002422.

9. Wallace BJ, Winchester JB, McGuigan MR. Effects of elastic bands on force and power characteristics during the back squat exercise. J Strength Cond Res. 2006; 20(2):268-272; doi: 10.1519/R-16854.1.

10. Weber KR, Brown LE, Coburn JW, Zinder SM. Acute effects of heavy-load squats on consecutive squat jump performance. J Strength Cond Res. 2008;22(3):726730; doi: 10.1519/JSC.0b013e3181660899.

11. Robbins DW. Postactivation potentiation and its practical applicability: a brief review. J Strength Cond Res. 2005;19(2):453-458; doi: 10.1519/R-14653.1.

12. Seitz LB, Haff GG. Factors modulating post-activation potentiation of jump, sprint, throw, and upper-body ballistic performances: a systematic review with meta- 
analysis. Sports Med. 2016;46(2):231-240; doi: 10.1007/ s40279-015-0415-7.

13. Jensen RL, Ebben WP. Kinetic analysis of complex training rest interval effect on vertical jump performance. J Strength Cond Res. 2003;17(2):345-349; doi: 10.1519/1533-4287(2003)017<0345:kaoctr>2.0.co;2.

14. Tillin NA, Bishop D. Factors modulating post-activation potentiation and its effect on performance of subsequent explosive activities. Sports Med. 2009;39(2): 147-166; doi: 10.2165/00007256-200939020-00004.

15. Kilduff LP, Owen N, Bevan H, Bennett M, Kingsley MIC, Cunningham D. Influence of recovery time on postactivation potentiation in professional rugby players. J Sports Sci. 2008;26(8):795-802; doi: 10.1080/02640 410701784517.

16. Gouvêa AL, Fernandes IA, César EP, Barbosa Silva WA, Chagas Gomes PS. The effects of rest intervals on jumping performance: a meta-analysis on post-activation potentiation studies. J Sports Sci. 2013;31(5):459467; doi: 10.1080/02640414.2012.738924.

17. Scott DJ, Ditroilo M, Marshall P. Effect of accommodating resistance on the postactivation potentiation response in rugby league players. J Strength Cond Res. 2018;32(9):2510-2520; doi: 10.1519/JSC.000000000 0002464

18. Seitz LB, Mina MA, Haff GG. Postactivation potentiation of horizontal jump performance across multiple sets of a contrast protocol. J Strength Cond Res. 2016; 30(10):2733-2740; doi: 10.1519/JSC.000000000000 1383.

19. Wallace BJ, Bergstrom HC, Butterfield TA. Muscular bases and mechanisms of variable resistance training efficacy. Int J Sports Sci Coach. 2018;13(6):1177-1188; doi: 10.1177/1747954118810240.

20. Cronin J, McNair PJ, Marshall RN. The effects of bungy weight training on muscle function and functional performance. J Sports Sci. 2003;21(1):59-71; doi: 10.1080/ 0264041031000071001.

21. Israetel MA, McBride JM, Nuzzo JL, Skinner JW, Dayne AM. Kinetic and kinematic differences between squats performed with and without elastic bands. J Strength Cond Res. 2010;24(1):190-194; doi: 10.1519/ JSC.0b013e31819b7995.

22. Jackson AS, Pollock ML. Generalized equations for predicting body density of men. Br J Nutr. 1978;40(3): 497-504; doi: 10.1079/bjn19780152.

23. LeSuer DA, McCormick JH, Mayhew JL, Wasserstein RL, Arnold MD. The accuracy of prediction equations for estimating 1-RM performance in the bench press, squat, and deadlift. J Strength Cond Res. 1997; 11(4):211-213; doi: 10.1519/00124278-19971100000001.

24. Raeder C, Wiewelhove T, Westphal-Martinez MP, Fernandez-Fernandez J, de Paula Simola RA, Kellmann $\mathrm{M}$, et al. Neuromuscular fatigue and physiological responses after five dynamic squat exercise protocols. J
Strength Cond Res. 2016;30(4):953-965; doi: 10.1519/ JSC.0000000000001181.

25. Beato M, Bigby AEJ, De Keijzer KL, Nakamura FY, Coratella G, McErlain-Naylor SA. Post-activation potentiation effect of eccentric overload and traditional weightlifting exercise on jumping and sprinting performance in male athletes. PLoS One. 2019;14(9): e0222466; doi: 10.1371/journal.pone.0222466.

26. Markovic G, Dizdar D, Jukic I, Cardinale M. Reliability and factorial validity of squat and countermovement jump tests. J Strength Cond Res. 2004;18(3):551-555; doi: 10.1519/1533-4287(2004)18<551:RAFVOS> 2.0.CO;2.

27. Sayers SP, Harackiewicz DV, Harman EA, Frykman PN, Rosenstein MT. Cross-validation of three jump power equations. Med Sci Sports Exerc. 1999;31(4):572-577; doi: 10.1097/00005768-199904000-00013.

28. Gołaś A, Maszczyk A, Zając A, Mikołajec K, Stastny P. Optimizing post activation potentiation for explosive activities in competitive sports. J Hum Kinet. 2016; 52:95-106; doi: 10.1515/hukin-2015-0197.

29. Baker DG. Increases in jump squat peak external power output when combined with accommodating resistance box squats during contrasting resistance complex training with short rest periods. J Aust Strength Cond. 2008;16(2):10-18.

30. Nikolaidis PT, Ingebrigtsen J. Physical and physiological characteristics of elite male handball players from teams with a different ranking. J Hum Kinet. 2013; 38:115-124; doi: 10.2478/hukin-2013-0051.

31. Strokosch A, Louit L, Seitz L, Clarke R, Hughes JD. Impact of accommodating resistance in potentiating horizontal-jump performance in professional rugby league players. Int J Sports Physiol Perform. 2018;13(9): 1223-1229; doi: 10.1123/ijspp.2017-0697.

32. Mola JN, Bruce-Low SS, Burnet SJ. Optimal recovery time for postactivation potentiation in professional soccer players. J Strength Cond Res. 2014;28(6):15291537; doi: 10.1519/JSC.0000000000000313.

33. Esformes JI, Cameron N, Bampouras TM. Postactivation potentiation following different modes of exercise. J Strength Cond Res. 2010;24(7):1911-1916; doi: 10.1519/JSC.0b013e3181dc47f8.

34. Gołaś A, Wilk M, Statsny P, Maszczyk A, Pajerska K, Zając A. Optimizing half squat postactivation potential load in squat jump training for eliciting relative maximal power in ski jumpers. J Strength Cond Res. 2017;31(11):3010-3017; doi: 10.1519/JSC.000000000 0001917. 